\title{
Disorder Effect on the Transmission of Second Harmonic Waves in One-Dimensional Periodically Poled $\mathrm{LiTaO}_{3}$
}

\author{
Bahrami Omid*, Baharvand Abdolrahim, Bahari Ali \\ Department of Physics, Faculty of Science, Lorestan University, Khorram-Abad, Iran \\ Email: *bahrami.om@fs.lu.ac.ir
}

How to cite this paper: Omid, B., Abdolrahim, B. and Ali, B. (2019) Disorder Effect on the Transmission of Second Harmonic Waves in One-Dimensional Periodically Poled $\mathrm{LiTaO}_{3}$. Journal of Modern Physics, 10, 432-442.

https://doi.org/10.4236/jmp.2019.104028

Received: February 19, 2019

Accepted: March 16, 2019

Published: March 19, 2019

Copyright ( 2019 by author(s) and Scientific Research Publishing Inc. This work is licensed under the Creative Commons Attribution International License (CC BY 4.0).

http://creativecommons.org/licenses/by/4.0/

\section{Open Access}

\begin{abstract}
One of the methods for calculating electromagnetic wave dispersion in multi-layer structures is the transfer matrix method. In this paper, we use the transfer matrix method for second harmonic generation in a nonlinear multilayer structure. The nonlinear photonic crystals investigated in this paper are as one-dimensional multi-layered structures including ferroelectric materials such as $\mathrm{LiTaO}_{3}$. Our goal is to investigate the effect of the disorder on the transmission spectrum of electromagnetic waves. Our results showed that positional disorder has different effects on the transmitting band and the gap band. The disorder in the transmitting band reduces the transmission coefficient of the waves and increases the transmission coefficient of the waves in the gap band. Such work has not yet been done on nonlinear photonic crystals producing the second harmonic.
\end{abstract}

\section{Keywords}

Transfer Matrix Method, Nonlinear Photonic Crystal, Random Binary

Medium, Transmission Spectra, Disorder Effect, Second Harmonic Waves

\section{Introduction}

In recent decades, the study of the transmission of waves in random systems has been highly studied because it plays an important role in understanding the optical, mechanical, magnetic, and electrical properties of materials. The most important theory in this case is Anderson's theory. This theory is about electrons crossing in random systems [1] [2]. Although Anderson's theory was initially about the transmission of electron waves in disordered systems, it was later extended to other waves such as electromagnetic and acoustic waves. 
A weak disorder in $3 \mathrm{D}$ devices does not turn all of the extended modes into localized modes. That is; there is a series of transmission modes in the system. For strong disorder, due to the incoherent interference of waves, transmitted modes are exponentially localized. For one-dimensional systems, Anderson's theory shows that all the passing modes are exponentially localized for any degree of disorder [2] [3].

There are several models for one dimensional multi-layer system in which exponential localization is violated. For example a one-dimensional system with the periodic period consisting of two parts is known as a random-dimer model [4] [5]. In this type of system, there are modes that disorder does not affect them. Another model that violates Anderson's localization in disordered one-dimensional systems is a long-range correlation model [6] [7]. The tight-binding $1 \mathrm{D}$ model also violates the exponential localization in one-dimensional systems that occur for any disorder [8] [9]. Finally, the last model that produces non-localized states, unlike the one-dimensional Anderson model prediction, is called the "necklace" model [10]. This model has been used in a multilayer dielectric structure [11].

A periodic structure with a transmission spectrum that does not have any propagating modes in certain frequency regions is called the photonic crystal [12]. Frequency spectrum, in which there are no propagating modes, is known as photonic band gap. Due to the vectorial nature of the electromagnetic waves, compared with the scalar nature of electron waves, the emission of electromagnetic waves in photonic crystals depends on its polarization and the wave propagation angle [13].

In a binary multi-layer structure made up of dielectric slabs, in which the optical lengths of the layers are equal, putting the disorder in the order of the layers leads to the appearance of necklace modes in the photonic band gap [14]. By placing a positional disorder in a multilayer structure, when the optical length of layer is equal to a quarter wavelength, a series of transmission modes through the photonic band gap are created [15].

Photonic crystals contain ferroelectric materials, due to the unique properties that compensate for the phase mismatch in the second harmonic generation, which have been much considered. Second harmonic generation is investigated in multilayer nonlinear structures including ferroelectrics such as $\mathrm{LiNbO}_{3}, \mathrm{KTi}$ $\mathrm{OPO}_{4}$ and strontium barium niobate [16] [17].

But until now, the effect of the disorder on the second harmonic generation (SHG) in the photonic crystals including ferroelectric materials has not been studied. We investigate the effects of positional disorder on the transmission spectrum of one-dimensional structures composed of lithium tantalite $\left(\mathrm{LiTaO}_{3}\right)$. We study a binary disordered system composed of $N$ periods. Using the transfer matrix method, we draw the transmission spectrum of the electromagnetic wave as a function of the incoming wave frequency. We study the effect of the positional disorder on the transmission of an electromagnetic wave that collides vertically on the surface of the structure, so that during the initial wave propagation 
in the nonlinear structure, a second harmonic wave is also produced.

This paper is organized as follows in Section 2 we focus on the details of the model and we derive the related transfer matrix. We also explain how we calculate the Transmission coefficient. In Section 3 we present and discuss our numerical results. Finally, Section 4 ends the paper with a summary of our results.

\section{Model and Method}

In this section, we study a multi-layered nonlinear structure, which changes the nonlinear parameter sign periodically in layers. In this structure, the scales of linear and nonlinear parameters are the same. The nonlinear structure is divided into $N$ periods of thickness $d$, and each period is divided into two homogeneous sections, Figure 1. Thickness and linear parameter (refractive index) and nonlinear parameter (second order susceptibility) for Section 1 are, respectively, $d_{I}$, $n_{I}^{(r)}$ and $\chi^{(2)}$, while those for Section 2 are, $d_{I I}, n_{I I}^{(r)}$ and $-\chi^{(2)}$, while $r=f, s$ is use for the fundamental field and second harmonic field, respectively. Due to the fact that the refractive index of regions $\mathrm{I}\left(n_{I}^{(r)}\right)$ and II $\left(n_{I I}^{(r)}\right)$ are not equal, its reflection will occur at each layer interface, resulting in between the transmitted and reflective waves in each layer, the phenomenon of interference will appear.

It is assumed that an electromagnetic wave with frequency $\omega$ incident from the left-hand side of the system and propagates in the direction of the $\mathrm{z}$ axis, so that the polarization of the electric field is in the $\mathrm{x}$-axis direction. Our numerical calculations are based on the nonlinear transfer matrix method (TMM) [18] [19]. In this method, the SHG process is divided into three stages: 1) First, the fundamental field (FF), with the propagation in the structure, causes the macroscopic polarization of matter. 2) Since the material is non-linear, then the second-order nonlinear polarization is created in matter. And this nonlinear polarization radiates the second harmonic ( $\mathrm{SH}$ ) field in the structure. 3) This second harmonic field is propagated in the device, and it comes out in the form of a second harmonic signal.

In the first stage of the second harmonic generation, the fundamental and second harmonic fields follow the following equations:

$$
\begin{aligned}
& \nabla \times\left(\nabla \times \boldsymbol{E}_{2 \omega}\right)-\frac{\varepsilon_{2 \omega}(2 \omega)^{2}}{c^{2}} \boldsymbol{E}_{2 \omega}=\mu_{0}(2 \omega)^{2} \boldsymbol{P}_{N L}^{2 \omega} \\
& \nabla \times\left(\nabla \times \boldsymbol{E}_{\omega}\right)-\frac{\varepsilon_{\omega} \omega^{2}}{c^{2}} \boldsymbol{E}_{\omega}=\mu_{0} \omega^{2} \boldsymbol{P}_{N L}^{\omega}
\end{aligned}
$$

In which, $\varepsilon_{\omega}, \varepsilon_{2 \omega}, \boldsymbol{P}_{N L}^{\omega}$, and $\boldsymbol{P}_{N L}^{2 \omega}$ are permittivity and nonlinear polarization at $F F$ and $S H$ frequencies. Because the nonlinear process is weak. As a result, the nonlinear process has no significant effect on the intensity of the fundamental pump field (Given that the material we have used in this paper is ferroelectric $\left(\mathrm{LiTaO}_{3}\right)$, whose second-order non-linear coefficient $\chi^{(2)}$ is $13.8 \mathrm{pm} / \mathrm{V}$. Therefore, in our calculations, we used the source approximation without decreasing (strong source approximation) to solve non-linear equations and 


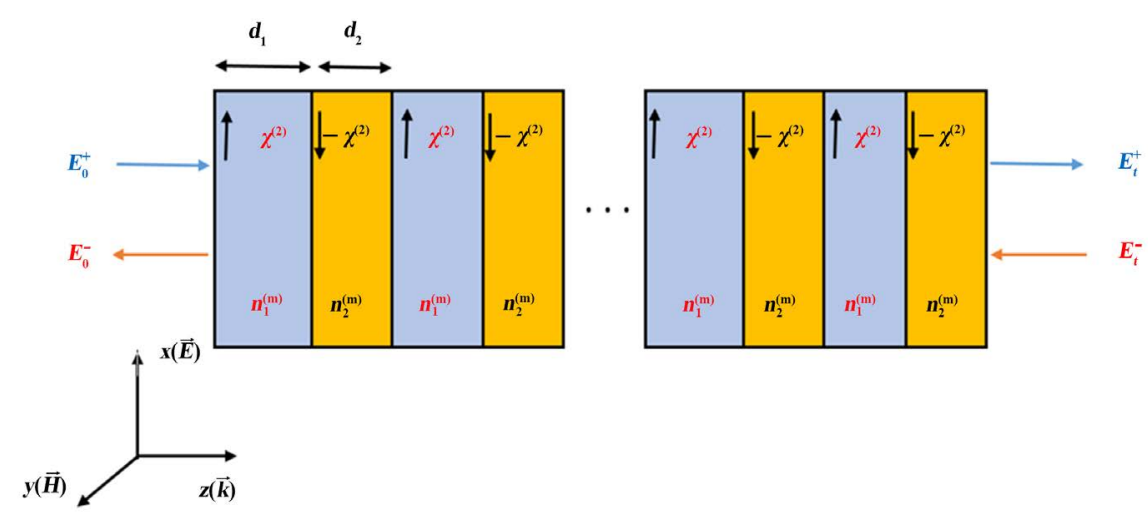

Figure 1. Multi-layered nonlinear structure. In each layer, the vectors represent the nonlinear polarization direction.

obtaining the transmission coefficient. That is, the second-order nonlinear coefficient is small, so the nonlinear process does not have a significant effect on the initial wave intensity). So here we are using the non-depleted pump wave approximation, $E_{2 \omega} \ll E_{\omega}$. Therefore, the above equations for the $m$-th layer are as follows

$$
\begin{aligned}
& {\left[\frac{\mathrm{d}^{2}}{\mathrm{~d}^{2}}+\left(k_{m}^{(f)}\right)^{2}\right] E_{m}^{f}(z)=0} \\
& {\left[\frac{\mathrm{d}^{2}}{\mathrm{~d} z^{2}}+\left(k_{m}^{(s)}\right)^{2}\right] E_{m}^{s}(z)=-\mu_{0}(2 \omega)^{2} \boldsymbol{P}_{N L}^{2 \omega}}
\end{aligned}
$$

where $k_{m}^{(f)}=n_{m}^{(f)} k_{0}^{(f)}, k_{m}^{(s)}=n_{m}^{(s)} k_{0}^{(s)}, k_{0}^{(f)}=\omega / c$, and $k_{0}^{(s)}=2 \omega / c$. and also, $n_{m}^{(f)}$ and $n_{m}^{(s)}$ show the refractive index of the pump waves and the second harmonic, respectively in the $m$ th slab; $c$, the speed of light is in vacuum

The first equation of coupling Equations (2) gives the fundamental electric field in the $m$ th layer.

$$
E_{m}^{(f)}=E_{m}^{f+} \mathrm{e}^{i\left(k_{m}^{(f)}\left(z-z_{m-1}\right)-\omega t\right)}+E_{m}^{f-} \mathrm{e}^{-i\left(k_{m}^{(f)}\left(z-z_{m-1}\right)-\omega t\right)}
$$

where $z_{0}$ is zero, $z_{m}=z_{m-1}+d_{m}$ and $d_{m}$ is the thickness of the $m$ th layer. $E_{m}^{(f)+}$ is the magnitude of forward plane wave and $E_{m}^{(f)-}$ is the magnitude of backward plane wave at the left interface of the $m$ th layer.

According to the continuity conditions on the boundary of the layers, we have the following matrix relation between the electric and magnetic fields passing through odd layer to even layer:

$$
\left(\begin{array}{c}
E_{2 m-1}^{f+} \\
E_{2 m-1}^{f-}
\end{array}\right)=\frac{1}{2 n_{I}^{(1)}}\left(\begin{array}{ll}
n_{I}^{(1)}+n_{I I}^{(1)} & n_{I}^{(1)}-n_{I I}^{(1)} \\
n_{I}^{(1)}-n_{I I}^{(1)} & n_{I}^{(1)}+n_{I I}^{(1)}
\end{array}\right)\left(\begin{array}{l}
E_{2 m}^{f+} \\
E_{2 m}^{f-}
\end{array}\right)=T_{12}\left(\begin{array}{c}
E_{2 i}^{f+} \\
E_{2 i}^{f-}
\end{array}\right)
$$

Using the matrix of dynamics and the matrix of propagation that are as follows.

$$
\begin{gathered}
D_{m}=\left(\begin{array}{cc}
1 & 1 \\
n_{m}^{(f)} & -n_{m}^{(f)}
\end{array}\right) \\
P_{m}=\left(\begin{array}{cc}
\exp \left(i k_{m}^{(f)} d_{m}\right) & 0 \\
0 & \exp \left(-i k_{m}^{(f)} d_{m}\right)
\end{array}\right)
\end{gathered}
$$


The general transfer matrix for the multi-layer structure is as follows

$$
T=D_{0}^{-1}\left(D_{I I} P_{I I} D_{I I}^{-1} D_{I} P_{I} D_{I}^{-1}\right)^{N} D_{0}
$$

This transfer matrix connects the fields at the beginning and the end of the structure, as follows

$$
\left(\begin{array}{l}
E_{t}^{f+} \\
E_{t}^{f-}
\end{array}\right)=T\left(\begin{array}{l}
E_{0}^{f+} \\
E_{0}^{f-}
\end{array}\right)
$$

For harmonic fields that is produced in the structure, we use Equation (2) with $P_{N L}^{2 \omega}(z, t)=\varepsilon_{0} \chi_{m}^{(2)}\left[E_{m}^{(f)}(z)\right]^{2} \exp (-i 2 \omega t)$ that yields to:

$$
\begin{aligned}
\left(\begin{array}{c}
E_{m}^{(s)+} \\
E_{m}^{(s)-}
\end{array}\right)= & G_{0}^{-1} S G_{0}\left(\begin{array}{c}
E_{m-1}^{(s)+} \\
E_{m-1}^{(s)-}
\end{array}\right)+G_{0}^{-1}\left(N_{I I} B_{I} F_{I}-S B_{I}\right) A_{I}\left(\begin{array}{c}
\left(E_{2 m-1}^{f+}\right)^{2} \\
\left(E_{2 m-1}^{f-}\right)^{2}
\end{array}\right) \\
& +G_{0}^{-1}\left(N_{I I}-S\right)\left(\begin{array}{c}
C_{I} E_{2 m-1}^{f+} E_{2 m-1}^{f-} \\
0
\end{array}\right) \\
& +G_{0}^{-1}\left(B_{I I} F_{I I}-N_{I I} B_{I I}\right) A_{I I}\left(\begin{array}{l}
\left(\Omega_{2 m}^{+}\right)^{2} \\
\left(\Omega_{2 m}^{-}\right)^{2}
\end{array}\right) \\
& +G_{0}^{-1}\left(I-N_{I I}\right)\left(\begin{array}{c}
C_{I I} E_{2 m}^{f+} E_{2 m}^{f-} \\
0
\end{array}\right)
\end{aligned}
$$

In which:

$$
\begin{gathered}
A_{l}=\frac{-4 \mu \varepsilon_{0} \chi_{l}^{(2)} \omega^{2}}{k_{l}^{(s) 2}-4 k_{l}^{(f) 2}}, C_{l}=\frac{-4 \mu \varepsilon_{0} \chi_{l}^{(2)} \omega^{2}}{k_{l}^{(s) 2}} \\
G_{l}=\left(\begin{array}{cc}
1 & 1 \\
n_{l}^{(s)} & -n_{l}^{(s)}
\end{array}\right), B_{l}=\left(\begin{array}{cc}
1 & 1 \\
\frac{2 k_{0}^{(f)} n_{l}^{(f)}}{k_{0}^{(s)}} & -\frac{2 k_{0}^{(f)} n_{l}^{(f)}}{k_{0}^{(s)}}
\end{array}\right) \\
Q_{i}=\left(\begin{array}{c}
\exp \left(i k_{l}^{(s)} d_{i}\right) \\
0 \\
\begin{array}{c}
0 \\
F_{l}=
\end{array} \\
\left.\begin{array}{c}
\exp \left(i 2 k_{l}^{(f)} d_{l}\right) \\
\left.0 \quad-i k_{l}^{(s)} d_{l}\right)
\end{array}\right) \\
S=G_{I I} Q_{I I} G_{I I}^{-1} G_{I} Q_{I} G_{I}^{-1}, N_{I I}=G_{I I} Q_{I I} G_{I I}^{-1}
\end{array}\right.
\end{gathered}
$$

In this section, we determined the method of obtaining the amplitude of the second harmonic field in the nonlinear multi-layer structure. We can not actually measure this quantity, but the intensity of the second harmonic is measurable (In this paper, which is a theoretical work, the intensity of the waves is obtained using the electromagnetic magnitude (intensity is proportional to the square of the field magnitude). The purpose of writing the phrase "measurable intensity of electromagnetic waves" is that in the laboratory, researchers measure the intensity of electromagnetic waves. In fact, this sentence is presented for 
comparison with laboratory work). Using the intensity of waves, we have the following definitions for the nonlinear reflectance and nonlinear transmittance:

$$
\begin{aligned}
R^{N L} & =\frac{I_{\text {harm }}^{r}}{\left(I_{\text {pump }}\right)^{2}} \\
T^{N L} & =\frac{I_{\text {harm }}^{t}}{\left(I_{\text {pump }}\right)^{2}}
\end{aligned}
$$

where $I_{\text {harm }}^{t, r}$ and $I_{\text {pump }}$ are the intensities of the reflected or transmitted harmonic and pump fields, respectively.

In our structure, it is assumed that the nonlinear photonic crystal are composed of periodically poled lithium tantalite $\left(\mathrm{LiTaO}_{3}\right)$ crystal, whose nonlinear susceptibility is $\chi^{(2)}=13.8 \mathrm{pm} / \mathrm{V}$ and The refractive index for lithium tantalite $\left(\mathrm{LiTaO}_{3}\right)$ is given by the following dispersion formula [20]:

$$
n^{2}(\lambda, T)=A+\frac{B+b(T)}{\lambda^{2}-[C+c(T)]^{2}}+\frac{E}{\lambda^{2}-F^{2}}+D \lambda^{2}
$$

where $A=4.5284, B=7.2449 \times 10^{-3}, C=0.2453, D=-2.3670 \times 10^{-2}$, $E=7.7690 \times 10^{-2}, \quad F=0.1838, \quad b(T)=2.6794 \times 10^{-8}(T+273.15)^{2}$, $c(T)=1.6234 \times 10^{-8}(T+273.15)^{2}$.

Periodic polar ferroelectric $\left(\mathrm{LiTaO}_{3}\right)$ crystals are described by modulations of the nonlinear susceptibilities. An optoelectronic effect is used to produce the photonic band gap structure. This effect is the origin of the modulation of the refractive index of the layers in the photonic crystal. If the electric field $E$ is applied in the direction of the optical axis of the material, correction of refractive index and new refractive index (in odd layers) are as follows:

$$
n_{1}=n+\Delta n_{1}=n-\frac{1}{2} n^{3} r_{33} E
$$

where $r_{33}$ is the optoelectronic coefficient of the material and $E$ is the electric field amplitude. Similar to the nonlinear optical coefficient, the optoelectronic coefficient of the material in different layers has different signs. So in reverse (even) layers the optoelectronic coefficient changes sign and the refractive index in these layers is written as follows:

$$
n_{2}=n+\Delta n_{2}=n+\frac{1}{2} n^{3} r_{33} E
$$

\section{Numerical Results}

We investigate a random binary structure constructed of $N$ lithium tantalite $\left(\mathrm{LiTaO}_{3}\right)$ layers. The positional disorder in the structure means the probability that the ith layer is Layer I or Layer II is equal. In order to have an overall picture of the role played by disorder, and to illustrate the effect of disorder in the nonlinear multi-layered structure, we compare the transmission spectrum of a periodic structure with alternating layers I and II with a disordered structure, as shown in Figure 2 for the structure with $N=70$ layers. 


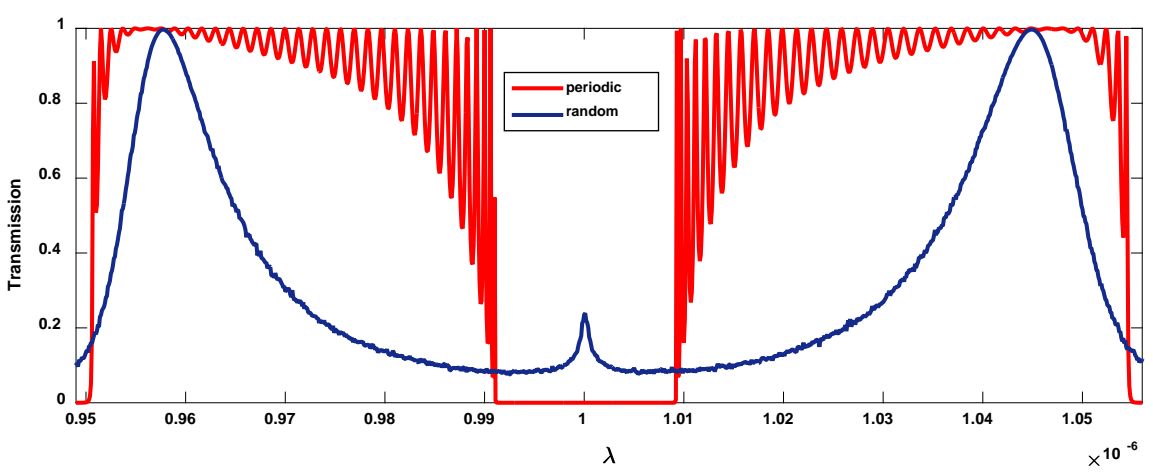

Figure 2. Transmission spectrum of periodic (red) and random (blue) binary structure with $N=70$ layers. For the random system, to calculate the transmission coefficient quantity, averaging operations are performed on 3000 systems with different ordering. The placement of disorder has different effects on the transmitting and gap bands. The disorder results in the appearance of transmission modes in the middle of the gap band.

The transmission spectrum for periodic structures is a sequence of transmitting and stop bands. In the transmitting band, there is a frequency in which the optical length of each layer is an integer multiple of half the wavelength in the vacuum. In other words, the phase change in each layer is equal to $m \pi$, that $m$ is the member of the integers. The nonlinear multi-layer structure for this incoming wave frequency is completely transparent. In the center of the stop bands, there are frequencies in which the optical length of each layer is odd multiples of one quarter wavelength. As a result, the phase change in these layers is equal to $(m+1 / 2) \pi$. For the random system, to calculate the Transmission coefficient quantity, averaging operations are performed on 3000 systems with different ordering (Figure 2). The placement of disorder has different effects on the transmitting and gap bands. Firstly, the disorder does not affect the frequency in the transmitting band in which the optical length of each layer is an integer multiple of half the wavelength. In other words, in a periodic and random structure at this frequency, the transmission coefficient is equal to the unit. Note that at this frequency, for each layer, the transfer matrix is a unit matrix (I). In this mode, independent of the existence and absence of disorder in the structure, is similar to the violation of Anderson localization in the random-dimer model. But, by placing disorder in the structure, the width of the transmission band is reduced. In the forbidden (gap) band, the disorder leads to the emergence of a series of transmitting modes. These mods are known as necklace modes.

In order to see the different effects of positional disorder in the transmitting band and the forbidden band, we plot the average transmission coefficient around the half- and quarter-wavelength modes as a function of the disorder intensity. In Figure 3 and Figure 4, $q$ shows the disorder strength, in other words, $q$ is equal to the probability that in the periodic structure (I, II, I, II, I, II, ... I, II), the ith layer, replaced with another layer. So $q=0$ is related to the periodic structure and $q=1 / 2$ is a structure with complete disorder. The results are shown in Figure 3 and Figure 4 for $N=70$. In order to obtain the transmission 


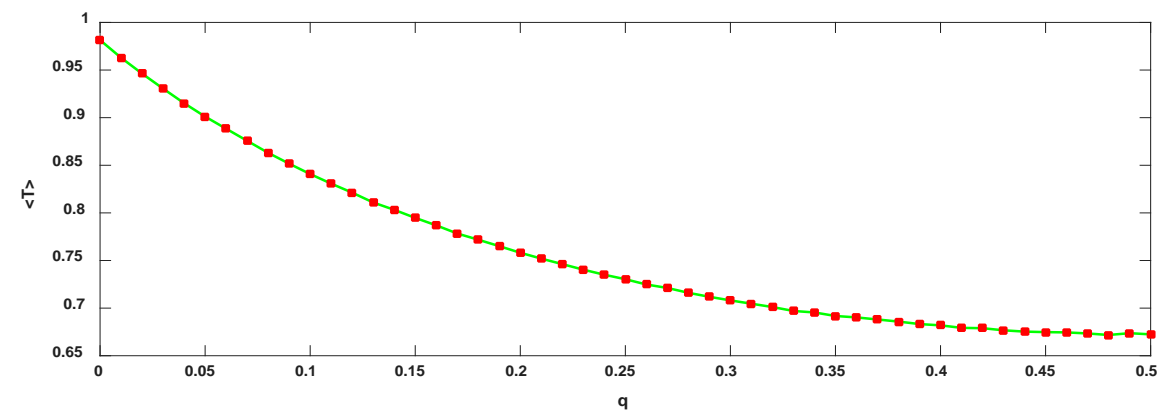

Figure 3. The average transmission coefficient as a function of the severity of the disorder, for the transmitting band. In this case, when the disorder increases, the transmission coefficient decreases, so Anderson's localization is established. The averaging is performed on 3000 structures in different order and $N=70$.

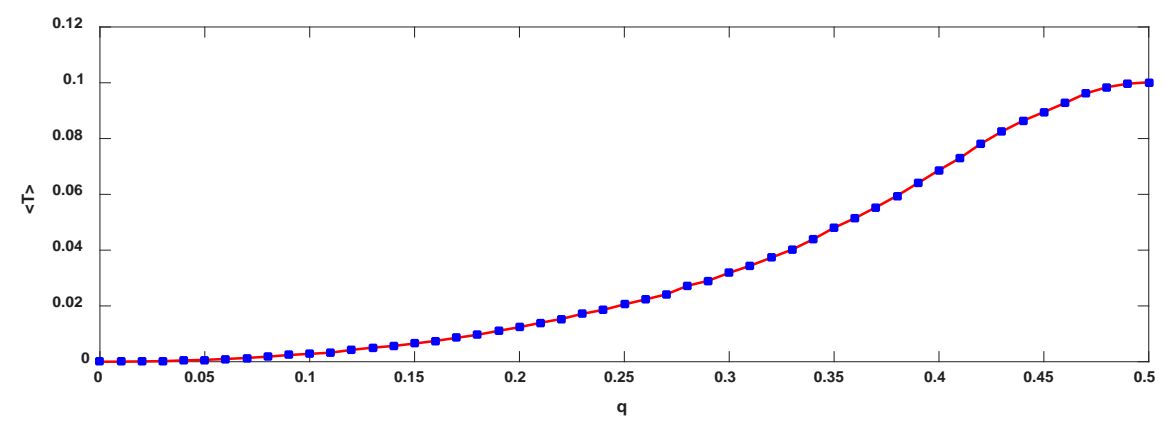

Figure 4. The average transmission coefficient as a function of the severity of the disorder, for the forbidden (gap) band. In quarter-wavelength modes, with increasing disorder, a series of transmitting modes are created, and thus a weak transmittance occurs at these frequencies. The averaging is performed on 3000 structures in different order and $N=70$.

coefficient in random structures, the averaging is performed on 3000 structures in different order. Figure 3 for the transmitting band and Figure 4 for forbidden band.

For the average transmission coefficient around the half-wavelength resonance, when the disorder increases, the transmission coefficient decreases, so Anderson's localization is established (Figure 3). Meanwhile, disorder effect in an around the quarter-wavelength resonance mode have a completely reverse result. As the disorder increases, the average transmittance increases (Figure 4). The reason for this is the appearance of transmitting modes in the forbidden band (Because in the periodic structure, in the gap band, the interference of the waves is non constructive. Using a disorder in the arrangement of the layers, the periodic structure that causes non-constructive interference of the waves and the transmission coefficient 0 in this frequency region (gaps) collapses. And with increasing disorder, the effect of the structure on the non-constructive interference of the waves decreases. As a result, the waves interact constructively. And there is a series of poorly transmitted modes in the gap band).

Figure 3 shows the average transmission coefficient in the transmitting band according to Anderson's localization. In this case, the average transmittance coefficient decreases monotonically with increasing intensity disorder. 


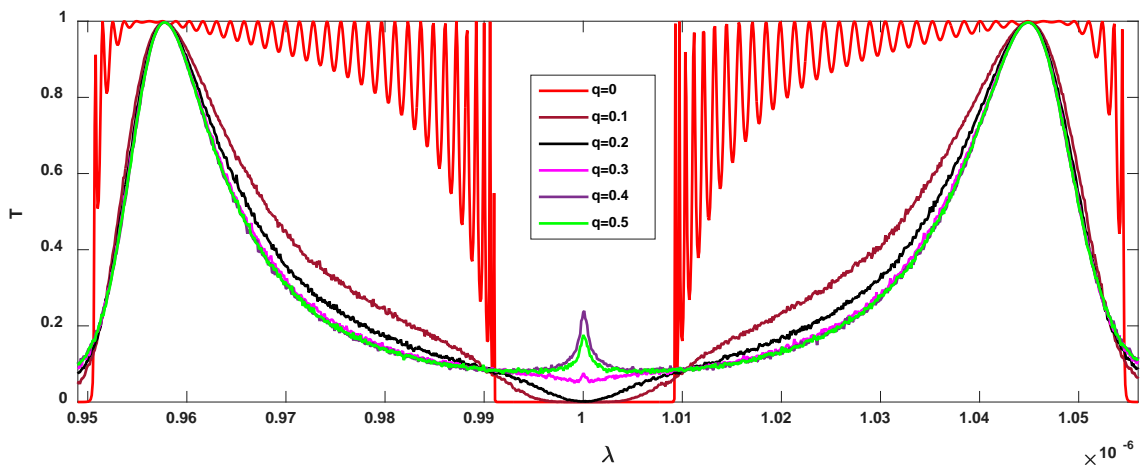

(a)

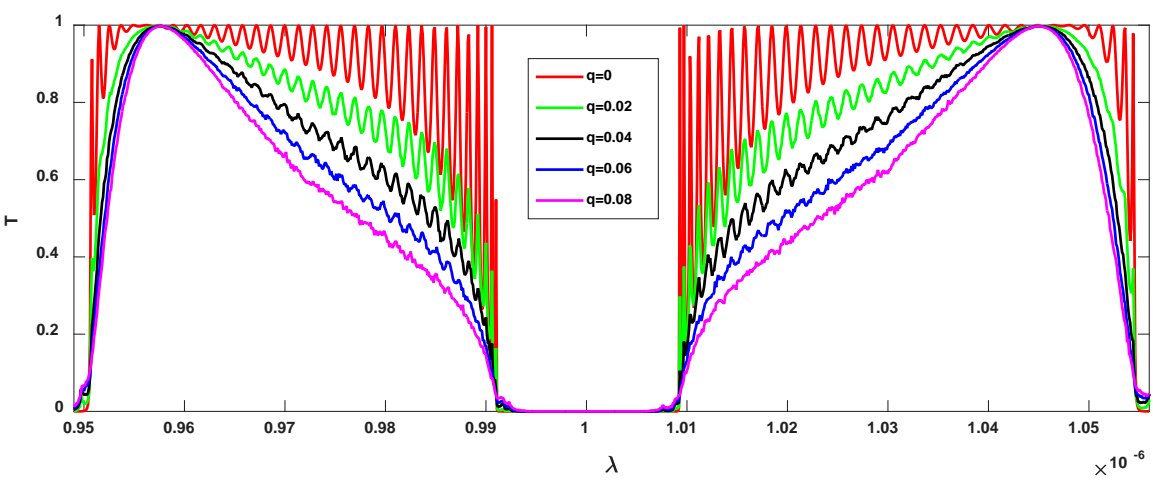

(b)

Figure 5. Transmission coefficient for different values of the disorder intensity. The number of layers is 70 and the averaging is performed on 2500 structures in different order. Figure 5(a) shows strong intensity disorder, in which case Anderson's theory is violated in the band gap. Figure 5(b) is for weak intensity disorders, in which case Anderson's localization is established.

While Figure 4 is a violation of Anderson's theory, the disorder result in the emergence of a few resonance necklace modes that creates a small transmittance in gap band.

Figure 5 shows the transmission coefficient as a function of the collision wavelength for different degrees of disorder. By increasing Severity of disorder, the bandwidth of transmission is reduced and a series of necklace mode is created in the gap bands which cause a slight increase in the transmission coefficient of electromagnetic waves. Figure 5(b) is for weak intensity disorders, in which case Anderson's localization is established. Figure 5(a) shows strong intensity disorder, in which case Anderson's theory is violated in the band gap.

\section{Results}

We studied the transmittance spectra in a nonlinear structure composed of tantalum lithium $\left(\mathrm{LiTaO}_{3}\right)$. This system is a binary structure with $N$ layer. The effect of disorder on the transmitting and forbidden bands is quite distinct. For the transmission band, the disorder in the frequency at which the coefficient of transmission is equal to 1 does not affect. At this frequency, the transfer matrix of each layer is equal to the unit matrix (I). The lack of sensitivity of this fully 
resonance mode is similar to the violation of Anderson's theory in the random-dimer model. However, the width of the transmittance band in a disordered system is narrower than the width of the transmittance band of the periodic system. For the gap band, disorder lead to the appearance of several modes in the center of the band. These modes are called necklace modes, which are based on the hybridization of degenerate modes localized. The transmittance in the stop (gap) band increases with the disorder intensity. The transmittance in the transmitting band decreases with the disorder intensity.

\section{Conflicts of Interest}

The authors declare no conflicts of interest regarding the publication of this paper.

\section{References}

[1] Anderson, P.W. (1958) Physical Review, 109, Article ID: 1492. https://doi.org/10.1103/PhysRev.109.1492

[2] Abrahams, E., Anderson, P.W., Licciardello, D.C. and Ramakrishnan, T.V. (1979) Physical Review Letters, 42, Article ID: 673. https://doi.org/10.1103/PhysRevLett.42.673

[3] Kramer, B. and MacKinnon, A. (1993) Reports on Progress in Physics, 56, 1469. https://doi.org/10.1088/0034-4885/56/12/001

[4] Dunlap, D.H., Wu, H.-L. and Phillips, P.W. (1990) Physical Review Letters, 65, Article ID: 88. https://doi.org/10.1103/PhysRevLett.65.88

[5] Phillips, P.W. and Wu, H.L. (1991) Science, 252, 1805-1812. https://doi.org/10.1126/science.252.5014.1805

[6] de Moura, F.A.B.F. and Lyra, M.L. (1998) Physical Review Letters, 81, Article ID: 3735 .

[7] Domínguez-Adame, F., Malyshev, V.A., de Moura, F.A.B.F. and Lyra, M.L. (2003) Physical Review Letters, 91, Article ID: 197402. https://doi.org/10.1103/PhysRevLett.91.197402

[8] Theodorou, G. and Cohen, M. (1976) Physical Review B, 13, Article ID: 4597. https://doi.org/10.1103/PhysRevB.13.4597

[9] Fleishman, L. and Licciardello, D.C. (1977) Journal of Physics C, 10, L125.

[10] Pendry, J.B. (1994) Advances in Physics, 43, 461-542. https://doi.org/10.1080/00018739400101515

[11] Bertolotti, J., Gottardo, S., Wiersma, D.S., Ghulinyan, M. and Pavesi, L. (2005) Physical Review Letters, 94, Article ID: 113903. https://doi.org/10.1103/PhysRevLett.94.113903

[12] Yablonovitch, E. (1987) Physical Review Letters, 58, Article ID: 2059. https://doi.org/10.1103/PhysRevLett.58.2059

[13] Fan, S.H., Villeneuve, P.R. and Joannopoulos, J.D. (1996) Physical Review B, 54, Article ID: 11245. https://doi.org/10.1103/PhysRevB.54.11245

[14] Sebbah, P., Hu, B., Klosner, J.M. and Genack, A.Z. (2006) Physical Review Letters, 96, Article ID: 183902. https://doi.org/10.1103/PhysRevLett.96.183902

[15] Bertolotti, J., Galli, M., Sapienza, R., Ghulinyan, M., Gottardo, S., Andreani, L.C., Pavesi, L. and Wiersma, D.S. (2006) Physical Review E, 74, Article ID: 035602. 
[16] Piskarskas, A., Smilgevicius, V., Stabinis, A., Jarutis, V., Pasiskevicius, V., Wang, S., Tellefsen, J. and Laurell, F. (1999) Optics Letters, 24, 1053-1055. https://doi.org/10.1364/OL.24.001053

[17] Zhu, Y.Y., Fu, J.S., Xiao, R.F. and Wong, G.K. (1997) Applied Physics Letters, 70, 1793-1795. https://doi.org/10.1063/1.118694

[18] Bell, P.M., Pendry, J.B., Marin Moreno, L. and Ward, A.J. (1995) Computer Physics Communications, 85, 306-322. https://doi.org/10.1016/0010-4655(94)00131-K

[19] Lin, L.L., Li, Z.Y. and Ho, K.M. (2003) Journal of Applied Physics, 94, 811-821. https://doi.org/10.1063/1.1587011

[20] Meyn, J.P. and Fejer, M.M. (1997) Optics Letters, 22, 1214-1216. https://doi.org/10.1364/OL.22.001214 